\title{
Influence of fluctuations of plasma large neutral amino acids with normal diets on the clinical response to levodopa
}

\author{
JOHN G NUTT, ${ }^{*} \dagger$ WILLIAM R WOODWARD, ${ }^{*} \ddagger$ JULIE H CARTER, ${ }^{*}$ \\ TRACI L TROTMAN* \\ From the Departments of Neurology,* Pharmacology, $\dagger$ and Biochemistry $\ddagger$, Oregon Health Sciences University, \\ Portland, Oregon, USA
}

SUMMARY Plasma large neutral amino acids (LNAAs) compete with levodopa for entry into the brain. Fluctuations in plasma LNAA concentrations could therefore contribute to variability in clinical response to levodopa. The hourly plasma levodopa, plasma LNAAs and clinical response were investigated in 11 fluctuating Parkinsonian patients on a regular hospital diet. The fluctuations in plasma levodopa were 2 to 3 times greater than the fluctuations of plasma LNAAs. The correlation between clinical response and plasma levodopa was substantially improved in only one patient by considering plasma LNAAs and calculating relative levodopa flux into brain. Although plasma LNAAs significantly increased during the day, the patients' clinical status did not uniformly deteriorate and mean afternoon clinical scores correlated better with mean plasma levodopa and levodopa flux than with mean plasma LNAAs. Minimum effective concentrations of levodopa for clinical response did not correlate with 9 am LNAA concentrations. It is concluded that in most patients, the relatively small variation in plasma LNAAs in comparison with the large variations in plasma levodopa indicates that fluctuations in LNAA are not an important contributor to the fluctuating response to levodopa.

Levodopa is transported from plasma into brain by the large neutral amino acid (LNAA) transport system of the cerebral capillary endothelial cells. ${ }^{1-3}$ At the normal plasma concentrations of LNAAs, this transport system is largely saturated such that there is competition between the various LNAAs for entry into brain. ${ }^{45}$ Influx of levodopa into brain is thus proportional to the plasma concentration of levodopa relative to the concentrations of the other LNAAs. Elevating plasma concentrations of LNAAs reduces the entry of levodopa into rat brain ${ }^{1-367}$ and human brain $^{8}$ and predictably, elevation of plasma LNAAs inhibits the clinical response to intravenously administered levodopa.' High protein diets decrease the response to levodopa and conversely low protein diets enhance the response to levodopa. ${ }^{10-18}$

\footnotetext{
Address for reprint requests: Dr J G Nutt, Department of Neurology, Oregon Health Sciences University, 3181 SW Sam Jackson Park Road, Portland, Oregon 97201, USA.
}

Received 7 June 1988 and in revised form 4 November 1988. Accepted 8 November 1988
Plasma amino acid concentrations fluctuate during the day in response to endogenous hormonal influences and to meals ${ }^{19-22}$ and these fluctuations affect brain concentrations of amino acids. ${ }^{2324}$ Moreover, each person appears to have a unique and characteristic plasma amino acid pattern. ${ }^{25}$ These fluctuations in LNAAs as well as the individual patterns of plasma amino acid concentrations could contribute to the fluctuating response to levodopa and determine each patient's sensitivity to levodopa.

We have investigated the hourly clinical response, plasma levodopa concentrations and plasma LNAA concentrations in 11 patients with Parkinson's disease to determine: (1) the variation in plasma LNAAs during the day on a regular hospital diet, (2) if the ratio of plasma concentrations of levodopa and LNAAs (levodopa flux) is a better predictor of clinical response than levodopa alone, (3) if plasma LNAA concentrations predict sensitivity to levodopa, and (4) if increasing plasma LNAA concentrations during the day contributes to the apparent afternoon worsening in some Parkinsonian patients. 


\section{Methods}

Subjects: Eleven patients ( 9 men and 2 women) with Parkinson's disease and a fluctuating response to levodopa (both predictable "wearing off" and seemingly unpredictable "onoff') participated in these studies after giving informed consent. Average age was 59 years (range 44 to 67), average disease duration 13 years (range 7 to 15 years) and average "off' Hoehn and Yahr stage 4 (range 3 to 5). All were taking levodopa with carbidopa (mean 1555 mg levodopa, range 80/ 800 to $500 / 2000$ [carbidopa/levodopa]). In addition, five were receiving dopamine agonists and five were receiving anticholinergics.

Protocol: Patients were hospitalised in the Clinical Research Center and clinical ratings and plasma samples obtained hourly from the first levodopa dose of the day (6 am to $8 \mathrm{am})$ until bedtime ( $10 \mathrm{pm}$ to 12 midnight). Diet was a standard hospital self-select diet with $15 \%$ of the calories as protein. Dietary intake was not monitored. Parkinsonian disability was scored by speed of tapping, speed of arising from a chair, walking a set distance and returning to the chair, and by scores for dyskinesia (positive) and tremor (negative) algebraically summed to give AIMs score.' Sensitivity to levodopa was equated with estimates of "threshold plasma levodopa concentrations" or "minimal effective concentrations" derived from levodopa infusion studies. ${ }^{26}$ These values were the first plasma levodopa concentrations recorded after the patient "turned on" during an infusion of levodopa at a constant rate ( 0 to 29 minutes after the clinical change). Threshold date was available for nine of the 11 patients described in this report and from an additional seven patients in whom only 9 am plasma LNAAs were measured. Levodopa and amino acid analyses: Levodopa was measured by high performance liquid chromatography with electrochemical detection. ${ }^{27}$ Plasma amino acids were measured by an HPLC procedure involving precolumn derivatisation with orthuphthaldehyde (OPA) and fluorescence detection. ${ }^{28} \mathrm{An}$ internal standard, $\beta$-aminobutyric acid (BABA, $15 \mathrm{nmol}$ ), was added to an aliquot of plasma $(100 \mu \mathrm{l})$, and proteins were precipitated by the addition of 4 volumes of ethanol. The precipitate was removed by centrifugation, and the supernatant was derivatised with OPA and fractionated by reversed-phase chromatography on a $\mathrm{C} 18$ column (Rainin, Microsorb; $5 \mathrm{~m}$ particle size, $5 \times 150 \mathrm{~mm}$ ) using a gradient elution from $20 \%$ methanol $/ 80 \mathrm{mM}$ sodium acetate to $70 \%$ methanol/60 mM sodium acetate $(\mathrm{pH} 7 \cdot 2)$. The intra-assay variability for amino acids was less than $5 \%$.

Data Analysis: The relative levodopa flux was calculated by an equation derived from Pardridge, ${ }^{4}$

$$
\text { Levodopa flux } \cong \frac{[\text { L-DOPA] }}{\sum \frac{\left[\text { LNAA }_{i}\right]}{\text { Km }_{i}}}
$$

where [L-DOPA] is plasma levodopa concentration, [LNAA] is plasma concentration of each LNAA and $\mathrm{Km}$ is the kinetic constant for that amino acid. The LNAAs used to calculate flux were tyrosine, phenylalanine, leucine, isoleucine and valine and their $\mathrm{Kms}$ derived from those measured in conscious rats. ${ }^{29}$

Because the clinical response often lags behind changes in plasma levodopa concentrations, ${ }^{26}$ we not only examined the relation between clinical response and concurrent plasma levodopa concentrations and fluxes, but also a "lagged" relation calculated by averaging the concurrent levodopa concentration or flux with the values from the preceding hour.

Linear, logarithmic and exponential regression analyses, paired $t$ tests, analysis of variance with repeated measures and least significant differences methods were used to assess significance as indicated in text and tables.

\section{Results}

\section{Diurnal variations in hourly plasma LNAAs and levodopa}

Standard deviations and ranges were used as estimates of variability of plasma levodopa and LNAA concentrations. The first plasma levodopa determination of the day was excluded from these calculations because it was invariably low if the patients had been without drug overnight. The standard deviation as a percent of the mean concentration averaged $17 \%$ for total plasma LNAAs and $43 \%$ for plasma levodopa. The range, as a percent of the mean concentration averaged $58 \%$ for LNAAs and $138 \%$ for levodopa. Thus variability in plasma levodopa concentrations was approximately two and one-half times that of total plasma LNAAs as measured by standard devia tions and by ranges (table 1 ).

\section{Correlation between clinical responses and levodopa concentrations of flux}

A significant linear correlation existed betweeñ levodopa concentrations or fluxes and hourly tapping scores in seven of 11 patients (fig 1, table 2); walking speed in nine of 11 patients (table 3); AIMs in seven of

Table 1 Mean hourly plasma large neutral amino acids and levodopa concentrations

\begin{tabular}{|c|c|c|c|c|c|c|}
\hline \multirow[b]{2}{*}{ Patients } & \multicolumn{3}{|c|}{ Plasma $L N A A s^{*}$} & \multicolumn{3}{|c|}{ Plasma levodopa $\dagger$} \\
\hline & Mean & $\begin{array}{l}S D \\
(\%) \ddagger\end{array}$ & $\begin{array}{l}\text { Range } \\
(\%) \S\end{array}$ & Mean & $\begin{array}{l}S D \\
(\%) \ddagger\end{array}$ & $\begin{array}{l}\text { Range } \\
(\%) \S\end{array}$ \\
\hline $\begin{array}{r}1 \\
2 \\
3 \\
4 \\
5 \\
6 \\
7 \\
8 \\
9 \\
10 \\
11\end{array}$ & $\begin{array}{l}496 \\
628 \\
658 \\
560 \\
710 \\
633 \\
525 \\
661 \\
599 \\
747 \\
548\end{array}$ & $\begin{array}{r}15 \\
17 \\
15 \\
12 \\
26 \\
22 \\
14 \\
11 \\
20 \\
9 \\
15\end{array}$ & $\begin{array}{l}55 \\
51 \\
47 \\
44 \\
98 \\
62 \\
45 \\
40 \\
79 \\
68 \\
51\end{array}$ & $\begin{array}{c}9.5 \\
6 \\
10.4 \\
14.2 \\
9.3 \\
7.4 \\
5.9 \\
9.4 \\
13.4 \\
10.7 \\
8.9\end{array}$ & $\begin{array}{l}39 \\
63 \\
43 \\
29 \\
40 \\
35 \\
38 \\
71 \\
41 \\
38 \\
33\end{array}$ & $\begin{array}{r}138 \\
168 \\
176 \\
148 \\
119 \\
111 \\
139 \\
261 \\
132 \\
128 \\
98\end{array}$ \\
\hline Mean & 615 & $17 \%$ & $58 \%$ & $9 \cdot 6$ & $43 \%$ & $138 \%$ \\
\hline
\end{tabular}

*Sum of concentrations of Phe, Tyr, Leu, Ile and Val in $\mathrm{nmol} / \mathrm{ml}$. tLevodopa in $\mathrm{nmol} / \mathrm{ml}$ excluding first determination of the day. tStandard deviation as percent of mean.

$\S$ Range as a percent of mean. Each patient mean represents 16 or 17 hourly measurements, except for patient 3 in whom only 12 observations were available. 
Table 2 Correlation between tapping scores and plasma levodopa and levodopa flux

\begin{tabular}{|c|c|c|c|c|}
\hline \multirow[b]{2}{*}{ Patient } & \multicolumn{4}{|c|}{ Linear correlation coefficients } \\
\hline & $L D \dagger$ & Lagged LD‡ & Flux§ & Lagged flux $\|$ \\
\hline $\begin{array}{r}1 \\
2 \\
3 \\
4 \\
5 \\
6 \\
7 \\
8 \\
9 \\
10 \\
11\end{array}$ & $\begin{array}{l}0.52^{*} \\
0.51^{*} \\
0.34 \\
0.36 \\
-0.29 \\
0 \cdot 25 \\
0 \cdot 30 \\
0.66^{* *} \\
0.81^{* * *} \\
0.54^{*} \\
0.81^{* * *}\end{array}$ & $\begin{array}{l}0.70^{* *} \\
0.65^{* *} \\
0.56 \\
0.44 \\
-0.11 \\
0.25 \\
0.41 \\
0.69^{* *} \\
0.83^{* * *} \\
0.50^{*} \\
0.20\end{array}$ & $\begin{array}{l}0.55^{*} \\
0.47 \\
0.39 \\
0.36 \\
0.07 \\
0.34 \\
0.33 \\
0.68^{* *} \\
0.73^{* *} \\
0.51^{*} \\
0.72^{* * *}\end{array}$ & $\begin{array}{l}0.74^{* * *} \\
0.69^{* * *} \\
0.58 \\
0.46 \\
0.40 \\
0.45 \\
0.46 \\
0.74^{* * *} \\
0.73^{* * *} \\
0.36 \\
0.24\end{array}$ \\
\hline
\end{tabular}

†Concurrent plasma levodopa concentration.

$\ddagger$ Average of preceding hour and concurrent plasma levodopa concentration.

§Concurrent levodopa fiux.

Average of preceding hour and concurrent levodopa fluxes.

${ }^{5}$ Correlation significant at $\mathrm{p}<0.05=*$
$\mathrm{p}<0.001=* * *$

the 10 patients with AIMs (data not shown) and mean sitting blood pressure in five of nine subjects in whom it was measured (data not shown). Subjects 3 and 7 had no significant correlation between any clinical score and levodopa or levodopa flux, although subject 3 had 12 rather than 16 or 17 measurements and just missed significance $(p=0.051)$. With the exception of patient 11 , an equal or better correlation was obtained with the average of the concurrent and previous hour levodopa or levodopa flux (lagged values) than with the concurrent values alone (tables 2 and 3 ). There was no significant difference between correlations on levodopa and on levodopa flux nor between correlations on lagged levodopa and on lagged levodopa flux (tables 2 and 3). In other words, considering the

Table 3 Correlation between walking times and plasma levodopa and levodopa flux

\begin{tabular}{lllll}
\hline & \multicolumn{4}{c}{ Linear correlation coefficients } \\
\cline { 2 - 5 } Patient & LD† & Lagged LD & Flux§ & Lagged flux\| \\
\hline 1 & -0.30 & $-0.65^{* *}$ & -0.33 & $-0.68^{* *}$ \\
2 & -0.26 & $-0.63^{* *}$ & -0.23 & $-0.66^{* *}$ \\
3 & -0.23 & -0.40 & -0.15 & -0.29 \\
4 & -0.31 & $-0.65^{* *}$ & -0.26 & $-0.68^{* *}$ \\
5 & -0.15 & -0.22 & -0.43 & $-0.67^{* *}$ \\
6 & $-0.50^{*}$ & $-0.75^{* * *}$ & -0.51 & $-0.71^{* * *}$ \\
7 & -0.17 & -0.37 & -0.20 & -0.40 \\
8 & $-0.56^{*}$ & $-0.59^{*}$ & $-0.62^{* *}$ & $-0.65^{* * *}$ \\
9 & $-0.76^{* * *}$ & $-0.87^{* * *}$ & $-0.70^{* *}$ & $-0.81^{* * *}$ \\
10 & -0.45 & -0.44 & -0.50 & $-0.49^{*}$ \\
11 & $-0.67^{* *}$ & -0.25 & $-0.58^{* *}$ & -0.08 \\
Mean & -0.40 & -0.53 & -0.41 & -0.56 \\
\hline
\end{tabular}

See table 2 for notation. Increasing walking score implies worsening Parkinsonism and thus the negative correlations. Lagged flux is significantly different $(p<0.05)$ from LD by ANOVA with repeated measures and differences between pairs of means evaluated by Least Significant Difference technique. plasma LNAAs by calculating relative flux did not significantly improve correlation between plasma levodopa and clinical response. Logarithmic or exponential regressions did not consistently or substantially improve on linear correlations (data not shown).

\section{Correlation between clinical response and plasma \\ LNAAs}

Hourly tapping, walking and AIM scores of subject 5 (fig 2) and AIM scores of subject 6 significantly correlated with the concurrent plasma LNAA concentrations. Subject 5 had the greatest variability in plasma LNAAs of all the patients and relatively low variability in plasma levodopa concentrations (table 1). Variability in LNAA concentrations of subject 6 also exceeded the mean of the group and variability in levodopa concentrations that was less than the mean (table 1).

\section{Comparison of morning and afternoon-evening plasma LNAAs and clinical response}

Plasma LNAAs increased an average of $19 \%$ during the day, from a mean of $550 \mathrm{nmol} / \mathrm{ml}$ for the morning hours (7 am to 12 noon) to $653 \mathrm{nmol} / \mathrm{ml}$ in the afternoon and evening (4 pm to $9 \mathrm{pm}$ ) (table 4 ). Levodopa tended to increase modestly in the afternoon as well and the consequence was that the calculated relative levodopa flux did not differ between morning and afternoon-evening for the group of patients as a whole (table 4). Likewise, there was no consistent difference between morning clinical scores (omitting the first score of the day) and the afternoonevening scores; some patients did better in the morning, some did better in the afternoon and some had a similar response in the morning and afternoon. Thus our group of patients did not substantiate the clinical impression of declining response to levodopa in the afternoons and evenings. However, the difference in morning and afternoon clinical scores did significantly correlate with the change in mean plasma levodopa between morning and afternoon-evening periods and to a lesser extent with changes in plasma LNAAs (table 5). In other words, the patients who did poorly in the afternoon and evening compared with the morning tended to have lower mean plasma levodopa concentrations and higher plasma LNAAs in the afternoon.

\section{Correlation of plasma LNAA concentrations to sensitivity to levodopa}

There was a two-fold difference in 9 am plasma LNAAs among patients and a four-fold difference in threshold levodopa concentrations. The 9 am LNAA concentrations did not correlate with the threshold or minimum effective concentrations of levodopa $(\mathrm{r}=0 \cdot 12, \mathrm{n}=16)$. 

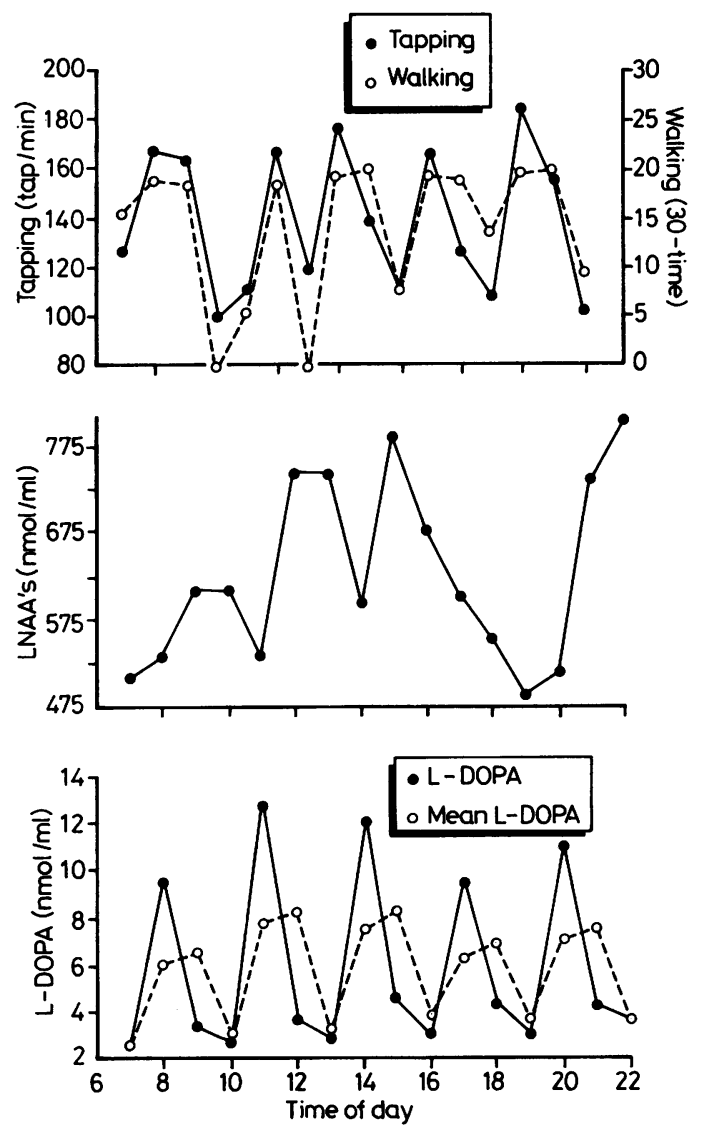

Fig 1 Levodopa, LNAA and clinical scores for a subject whose motor performance was largely determined by levodopa concentrations (patient 2). The bottom panel illustrates the plasma levodopa concentrations (dark line) and the lagged or running mean (average of concurrent and preceding hour concentrations) (light line). The top panel illustrates the tapping speed (dark line) and walking speed (light line). Walking speed is represented as $30 \mathrm{~s}$ minus the walking time (larger values on graph represent faster walking and better mobility). Clinical scores correlated better with lagged levodopa than levodopa and were not improved by calculating flux (tables $2 \& 3$ ). There was no correlation between clinical scores and plasma LNAA $(r=0 \cdot 10$ for both scores).

\section{Discussion}

Because of the competition between LNAAs and levodopa for transport across the blood-brain barrier, we postulated that diurnal variations in LNAA levels might be responsible for some apparently unpredictable clinical fluctuations during the day ("on-off" phenomenon), afternoon worsening and apparent sensitivity to levodopa. Our data, however, suggest that for most patients on normal diets, plasma amino acids are not a major determinant of clinical response for the reasons below.

First, the variability in plasma LNAAs in Parkinson's disease patients on a self-select hospital diet was similar to that reported for normal individuals, ${ }^{25}$ but was small compared with the marked swings in plasma levodopa concentrations. Thus, if entry of levodopa into brain is proportional to the ratio of levodopa to LNAAs then most of the variability in flux will be a result of the variability in plasma levodopa. This was confirmed by regression analysis; plasma levodopa
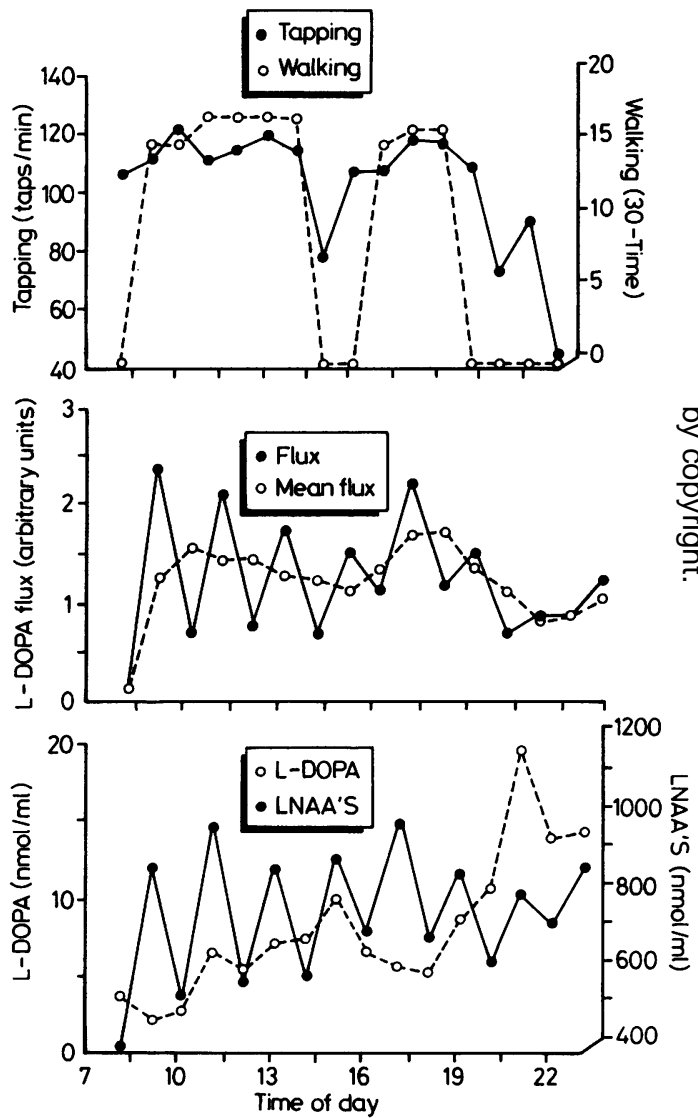

Fig 2 Levodopa, LNAA, levodopa flux and clinical scores for the subject whose clinical performance was largely influenced by plasma LNAAs (patient 5). The bottom panel illustrates plasma levodopa (dark line) and LNAAs (light line) concentrations. The middle panel illustrates levodopa flux (dark line) and mean or lagged flux (light line). The top panel illustrates clinical scores. Clinical scores did not correlate with levodopa or mean levodopa but did correlate with mean flux (tables 2 \& 3) and with LNAAs $(r=-0.74$ for tapping and $r=-0.60$ for walking). 
Table 4 Diurnal patterns of LNAAs, levodopa, flux and clinical scores

\begin{tabular}{lll}
\hline & \multicolumn{2}{l}{ Afternoon-Morning } \\
\cline { 2 - 3 } Parameter & Difference* & Probability $\dagger$ \\
\hline LNAA (nmol/ml) & 103 & 0.01 \\
Levodopa (nmol/ml) & 1.3 & 0.1 \\
Flux (arbitrary units) & -0.02 & 0.9 \\
Tapping (taps/min) & 1.6 & 0.8 \\
Walking (sec) & -2.3 & 0.3 \\
AIMS (arbitrary units) & 1.0 & 0.4 \\
\hline
\end{tabular}

* Mean of differences (afternoon-evening averages minus morning averages)

$\uparrow$ Probability that difference is significant by paired $t$ test.

concentrations were highly correlated with relative levodopa flux (mean of correlation coefficients from each patient $=0.95$, SD 0.03 ) and poorly correlated with plasma LNAAs $(r=0.32, \mathrm{SD} 0.15)$. This implies that plasma amino acids contribute $10 \%$ of the variability in the levodopa flux into brain and plasma levodopa $90 \%$. Ericksson et $a l^{30}$ reached similar conclusions.

Second, the correlation between clinical ratings and plasma levodopa concentrations (concurrent or average of concurrent and previous hour values) was not significantly improved by considering the plasma LNAAs and calculating levodopa flux in 10 of the 11 patients. It is pertinent that patient 5 , in whom flux was an appreciably better predictor of clinical response, had the largest fluctuations in plasma LNAAs. Nevertheless, we conclude that fluctuations in plasma LNAAs during ingestion of a regular hospital diet are generally not large enough to be a major contributor to the fluctuating response.

These results do not contradict other studies showing that very large amino acid loads reduce levodopa entry into brain ${ }^{1-36-8}$ and the clinical response. 9 Two- to four-fold elevations of plasma LNAAs were observed in these human studies, ${ }^{89}$ elevations which are much larger than those we measured during consumption of regular hospital diets. Our results do not directly address the question of the effect of low and high

Table 5 Correlation between differences in morning and afternoon clinical scores and changes in plasma LNAAs, levodopa and flux

\begin{tabular}{lccr}
\hline & \multicolumn{3}{c}{ Linear correlation coefficients } \\
\cline { 2 - 4 } Clinical parameter & LNAAs & Levodopa & \multicolumn{1}{l}{ Flux } \\
\hline Tapping & $-0.68^{*}$ & $0.62^{*}$ & $0.74 \dagger$ \\
Walking & 0.56 & $-0.69^{*}$ & $-0.62^{*}$ \\
AIMS & -0.43 & $0.67^{*}$ & $0.62^{*}$ \\
\hline
\end{tabular}

Increasing Tapping and AIMS scores and decreasing Walking scores imply improvement in Parkinsonism. Improvement is negatively correlated with increasing LNAAs and positively correlated with increasing levodopa. ${ }^{*} p<0.05,+p<0.01$. protein diets on plasma amino acids and clinical response. However, subjects 1,8 , and 9 subsequently participated in a dietary study comparing $1.6 \mathrm{~g}$ protein $/ \mathrm{kg}$ body weight/day with a $0.8 \mathrm{~g} / \mathrm{kg} \mathrm{diet}{ }^{18}$ and the same dependence of hourly scores on plasma levodopa rather than LNAA concentrations persisted although the patients had more "on" time with low protein diets. Protein content of the diet thus seems to affect sensitivity or response threshold to levodopa but plasma levodopa remains the prime determinant of the minute to minute clinical response.

The $19 \%$ rise in plasma amino acids in the afternoon was statistically significant but accompanied by a rise in plasma levodopa so that there was no alteration in levodopa flux. This was reflected in no significant change in the mean of the afternoon clinical scores in our 11 patients. However, the three patients who clearly worsened in the afternoon and evening were those with the greatest increase in plasma LNAAs (mean $42 \%$ increase) while their levodopa concentrations remained relatively stable (mean $6 \%$ decrease). Thus, some afternoon worsening may be related to increasing plasma LNAAs during the day in response to protein intake. Nevertheless, the differences between morning and afternoon clinical performance correlated better with the differences in levodopa concentrations between morning and afternoon-evening than with the differences in LNAA concentrations.

Threshold plasma levodopa concentrations for clinical response determined during infusion studies are crude estimates because there is a lag between elevation of plasma levodopa and the clinical response and this lag will depend somewhat upon the rate of infusion. Furthermore, levodopa and clinical ratings are done at half-hour intervals, reducing precision. However, these estimates do give some indication of a patient's sensitivity to levodopa, differing four-fold among patients, and generally correlated with the patient's daily levodopa consumption $(\mathrm{mg} / \mathrm{kg} / \mathrm{day})$. The minimum effective levodopa concentrations did not correlate with 9 am plasma LNAA concentrations, suggesting that this difference in sensitivity to levodopa among patients is not largely determined by plasma LNAAs competing for transport with levodopa at the blood brain barrier.

Would consideration of other LNAAs not used in our calculations of flux, specifically histidine, methionine, tryptophan and 3-0-methyldopa, alter our conclusions? Probably not. Histidine has a relatively low affinity for the LNAA carrier ${ }^{29}$ and methionine is present in very low concentrations. Moreover, the plasma concentrations of both these amino acids move in concert with other LNAAs ${ }^{14}$ and thus their inclusion in the calculations of relative levodopa flux would not substantially change the calculated pattern of levodopa flux. Plasma tryptophan is largely protein 
bound (and thus largely unmeasurable by our assay) and its role as a competitor for transport is uncertain. The levodopa metabolite, 3-0-methyldopa, also uses the LNAA transport system, but concentrations of this LNAA are both relatively low and constant throughout the day, and, as we have argued elsewhere, are unlikely to contribute much variability to the relative flux of levodopa. ${ }^{31}$

A disturbing feature of our results is the poor linear correlation between plasma levodopa or levodopa flux and clinical response. For example, the mean of the largest regression coefficients obtained by regressing walking times on concurrent and lagged levodopa or levodopa fluxes suggests that only $42 \%$ (range $16-$ $76 \%$ ) of the variability of walking times is accounted for by levodopa or levodopa flux. In most patients averaging the concurrent and previous hour levodopa concentrations or fluxes yielded better correlations, probably representing a correction for the lag or hysteresis between changes in plasma levodopa and clinical response. ${ }^{26}{ }^{30}$ It may be that there would be a better correlation between clinical ratings and plasma levodopa measured 20 to 40 minutes prior to the clinical rating. This lag may also vary between patients and represent another pharmacokinetic or pharmacodynamic variable, similar to plasma clearances and half-lives.

Another explanation for the relatively poor correlation between plasma levodopa concentration and clinical response is that the relationship between plasma levodopa and clinical response is not linear. We have previously noted that the response in fluctuating patients is largely "all or nothing" ${ }^{26}$ and many pharmacodynamic models suggest a logarithmic or exponential relationship between drug concentrations and response. Logarithmic and exponential regressions, however, did not significantly improve the correlation coefficients. Even more complicated doseresponse relationships such as biphasic response with low concentrations of dopaminergic agents worsening mobility and higher concentrations improving mobility ${ }^{32}$ may further distort the correlation. Finally, repeated dosing during the day may acutely alter sensitivity to the drug.

The clinical implication of these studies is that trying to adjust meals to reduce fluctuations in plasma LNAAs will rarely reduce the fluctuations in response to levodopa. Indeed, in four patients in whom we compared clinical response with a diet given as six evenly spaced meals and as three evenly spaced meals there was no clinical advantage to the multiple feedings, a result consistent with others' experience. ${ }^{13} \mathrm{On}$ a more theoretical level, the poor linear correlation between plasma levodopa and clinical response presents a challenge to discover the other factors that influence clinical response and to develop phar- macologic models that better predict the moment to moment response to levodopa.

We thank the patients for participating in demanding studies, the nurses of the Clinical Research Center for careful execution of protocols and empathetic care of patients, and Ana Gage and Laila Wilby for preparation of the manuscript. Supported by NIH-NINCDS R01 NS21062-04 and Clinical Research Center grant RR 00334.

\section{References}

1 Oldendorf WH. Brain uptake of radiolabeled amino acids, amines, and hexoses after arterial injection. Am J Physiol 1971; 221:1629-39.

2 Wade LA, Katzman R. Synthetic amino acids and the nature of $L$ DOPA transport at the blood-brain-barrier. $J$ Neurochem 1975;25:837-42.

3 Daniel PM, Moorhouse SR, Pratt OE. Do changes in blood levels of other aromatic amino acids influence levodopa therapy? Lancet 1976;i:95.

4 Pardridge WM. Kinetics of competitive inhibition of neutral amino acid transport across the blood-brain-barrier. J Neurochem 1977;28:103-8.

5 Pardridge WM, Choi TB. Neutral amino acid transport at the blood-brain-barrier. Fed Proc 1986;45:2073-8.

6 Gervas JJ, Muradas V, Bazan E, Aguado EG, deYebenes JG. Effects of 3-0-methyldopa on monoamine metabolism in rat brain. Neurology 1983;33:278-82.

7 Reches A, Fahn S. 3-0-methyldopa blocks dopa metabolism in raf corpus striätum. Ann Neurol 982;12:267-71.

8 Leenders KL, Poewe WH, Palmer AJ, Brenton DP, Frackowiak RSJ. Uptake into human brain by amino acids demonstrated by positron emission tomography. Ann Neurol 1986;20:258-62.

9 Nutt JG, Woodward WR, Hammerstad JP, Carter JH, Anderson JL. The "on-off' phenomenon in Parkinson's disease: Relation to levodopa absorption and transport. N Engl J Med 1984; 310:483-8.

10 Mena I, Cotzias GC. Protein intake and treatment of Parkinson's disease with levodopa. N Engl J Med 1975;292:131-84.

11 Sweet RD, McDowell FH. Plasma dopa concentrations and the "on-off" effect after chronic treatment of Parkinson's disease. Neurology 1974;24:953-6.

12 Pincus JH, Barry $\mathrm{K}$. Influence of dietary protein on motor fluctuations in Parkinson's disease. Arch Neurol 1987;44:270-2.

13 Juncos JL, Fabbrini G, Mouradian MM, Serrati C, Chase TN. Dietary influences on the antiparkinsonian response to levodopa. Arch Neurol 1987;44:1003-5.

14 Pincus JH, Barry K. Protein redistribution diet restores motor function in patients with dopa-resistant "off" periods Neurology 1988;38:481-3.

15 Riley D, Lang AE. Practical application of a low protein diet for Parkinson's disease. Neurology 1988;38:1026-31.

16 Eriksson T, Grarierus A-K, Linde A, Carlsson A. "On-off" phenomenon in Parkinson's disease: Relation between dopa and other large neutral amino acids in plasma. Neurology 1988;38:1245-8.

17 Tsui JKC, Ross S, Poulin K, et al. The effect of dietary protein on the efficacy of L-DOPA: A double blind study. Neurology 1989 (in press.)

18 Carter JH, Nutt JG, Woodward WR, Hatcher L, Trotman T. Amount and distribution of dietary protein affects clinical response to levodopa. Neurolopgy 1989 (in press.)

19 Wurtman RJ, Rose CM, Chow C, Larin FF. Daily rhythms in the 
concentrations of various amino acids in human plasma. $N$ Engl J Med 1968;279:171-5.

20 Fernstrom JD, Wurtman RJ, Hammarstrom-Wirlund B, Rand WM, Munro HN, Davidson CS. Diurnal variations in plasma concentrations of tryptophan, tyrosine, and other neutral amino acids: Effect of dietary protein intake. Am J Clin Nutr 1979;32:1912-22.

21 Frame EG. The levels of individual free amino acids in the plasma of normal men at various intervals after a high protein meal. $J$ Clin Invest 1958;37:1710-23.

22 Moller SE. Effects of various oral protein doses on plasma neutral amino acids. J Neurol Transmission 1985;61:183-91.

23 Fernstrom JD, Faller DV. Neutral amino acids in the brain: changes in response to food ingestion. $J$ Neurochem 1978; 30:1531-8.

24 Glaeser BS, Maher TJ, Wurtman RJ. Changes in brain levels of acidic, basic and neutral amino acids after consumption of single meals containing various proportions of protein. $J$ Neurochem 1983;41:1016-21.

25 Scriver CR, Gregory DM, Sovetts D, Tissenbaum G. Normal plasma free amino acids values in adults: The influence of some common physiological variables. Metabolism 1985;34:868-73.

26 Nutt JG, Woodward WR. Levodopa pharmacokinetics and pharmacodynamics in fluctuating parkinsonian patients.
Neurology 1986;36:739-44.

27 Nutt JG, Woodward WR, Carter JH. Clinical and biochemical studies with controlled-release Sinemet. Neurology 1986;36: 1206-11.

28 Jones BN, Gilligan JP. O-Phthaldialdehyde precolumn derivatiation and reversed-phase high-performance liquid chromatography of polypeptide hydrolysates and physiological fluids. $J$ Chromatogr 1983;266:471-82.

29 Miller LP, Pardridge WM, Braun LD, Oldendorf WH. Kinetic constants for blood-brain-barrier amino acid transport in conscious rats. J Neurochem 1985;45:1427-32.

30 Ericksson T, Magnusson T, Carlsson A, Linde A, Granerus AK. "On-off" phenomenon in Parkinson's disease: Correlation to the concentration of DOPA in plasma. J Neural Transmission 1984;59:229-40.

31 Nutt JG, Woodward WR, Gancher ST, Merrick D. 3·0'methyldopa and the response to levodopa in Parkinson's disease. Ann Neurol 1987;21:584-8.

32 Paalzow GHN, Paalzow LK. L-DOPA: How it may exacerbate parkinsonism symptoms. Trends in Pharmacological Sciences 1986;9:15-19.

33 Nutt JG, Gancher ST, Woodward WR. Does an inhibitory action of levodopa contribute to "on-off'? Neurology 1988;38: 1553-7. 